06

\title{
Магнитогидродинамический принцип 3D-печати для расплавов цветных металлов
}

\author{
() В.Б. Ошурко, А.М. Мандель, Е.Е. Карпова, А.А. Шарц, К.Г. Соломахо \\ Московский государственный технологический университет „СТАНКИН“, \\ 127055 Москва, Россия \\ e-mail: kirgeosol@gmail.com
}

Поступило в Редакцию 15 ноября 2017 г.

В окончательной редакции 1 ноября 2018 г.

Принято к публикации 1 ноября 2018 г.

Предложен новый принцип высокоэффективной $3 D$-печати для расплавов цветных металлов. Температура кристаллизации может повышаться в нижней части сужающейся струи жидкого металла за счет давления, создаваемого силами Ампера, вызванными специально приложенным током. Изучен вопрос о принципиальной возможности такой кристаллизации с учетом дополнительного нагрева, создаваемого этим током. Обнаружено, что введение ферромагнитных добавок позволяет создать область параметров, в которых такая кристаллизация возможна.

DOI: $10.21883 / J T F .2019 .05 .47471 .2557$

\section{Введение}

В настоящее время аддитивные технологии привлекают большое внимание, хотя, за исключением случая расплавов полимеров, их возможности оставляют желать лучшего. Так, для лазерного спекания характерные производительности редко превышают величину в несколько десятков граммов в час, для холодного напыления - десятков граммов в минуту. При этом качество синтезированного материала также оказывается низким. Эти процессы исследуются уже более двух десятилетий, так что, по-видимому, возможности их оптимизации почти исчерпаны. В связи с этим появляется интерес к принципиально новым методам трехмерной печати, в частности, для задач машиностроения. В настоящей работе анализируется такая возможность создания высокоэффективного $3 D$-принтера для жидких металлов. Суть метода состоит в следующем. Как известно, при пропускании тока через струю расплавленного металла возникают силы Ампера, приводящие к сжатию проводника. С другой стороны, температура кристаллизации для большинства металлов растет с ростом давления [1]. Если в области конца струи создать достаточно большое давление, то в этой области температура жидкого металла окажется ниже точки кристаллизации. Очевидно, что этим процессом можно управлять путем изменения протекающего тока и, при создании соответствующих процедур $2 D$-сканирования и удаления излишков расплава осуществлять трехмерную печать изделий с производительностью, определяемой только скоростью потока расплава.

Однако при этом возникает целый ряд вопросов, способных существенно повлиять на саму возможность реализации такой печати. Так, увеличение тока, необходимое для создания давления, одновременно должно вызывать дополнительный нагрев, препятствующий кри- сталлизации, и т. п. Пока совершенно неясно, существует ли вообще такая область параметров, в которой в принципе достижим описанный тепловой режим струи „принтера“. Иначе говоря, необходимо выяснить, может ли указанное повышение температуры кристаллизации превалировать над нагревом током.

Итак, цель настоящей работы - построение магнитогидродинамической модели истечения струи расплава металла при протекании через нее тока с учетом баланса тепла для выяснения принципиальной возможности новой аддитивной технологии описанного типа.

\section{Магнитогидродинамическая модель}

Рассмотрим струю жидкого металла, истекающую из резервуара вдоль оси $z$ (направленной вниз) с начальной скоростью $v_{0}$. Будем считать режим истечения устоявшимся, так что $d \vec{v} / d t=0$, и можно использовать обычное стационарное уравнение магнитогидродинамики уравнение Навье-Стокса с магнитным членом [2]:

$$
(\vec{v} \nabla) \vec{v}=\nu \Delta \vec{v}-\frac{1}{\rho} \nabla p+\vec{g}+\frac{1}{\rho}[\vec{j}, \vec{B}],
$$

где $\vec{v}-$ скорость, $p$ - давление, $v-$ вязкость, $g$ ускорение свободного падения, $\vec{j}-$ плотность тока и $\vec{B}$ - магнитное поле.

Прежде всего, заметим, что в нашем случае магнитное поле не является внешним, а задается самим током, протекающим по струе. Оценим величину этого магнитного поля, стандартно полагая плотность электрического тока постоянной по сечению струи ${ }^{1}$. С помощью теоремы о

\footnotetext{
${ }^{1}$ Строго говоря, распределение плотности тока по сечению струи определяется из условия минимизации энергии (например, [3]). Это, однако, может изменить нашу оценку лишь на постоянный коэффициент порядка единицы.
} 
циркуляции, выбирая контур в виде кольца радиуса $r$ внутри струи $(r<R)$, получаем для распределения индукции магнитного поля по радиусу струи

$$
B(r)=\mu \mu_{0} j r / 2
$$

где плотность тока

$$
j=I / \pi R^{2}(z)
$$

Здесь $\mu$ - магнитная проницаемость жидкого металла, $\mu_{0}$ - магнитная постоянная, $I-$ сила тока. Тогда в цилиндрических координатах последний член в уравнении (1) имеет лишь радиальную компоненту, равную

$$
-\frac{\mu \mu_{0}}{\rho} r j^{2}
$$

и представляющую собой силу Ампера (отнесенную к плотности $\rho$ ), действующую, как и следует, вдоль радиуса и направленную к оси струи, т.е. сжимающую ее. Далее будем полагать, что режим течения струи является практически ламинарным, а диаметр струи много меньше ее длины. Ясно также, что угловой компонент скорости потока мы можем принять за нуль (угловое движение струи могут вызвать лишь случайные факторы) $v_{\phi}=0$, а радиальный компонент скорости пренебрежимо мал по сравнению с осевым $v_{r} \ll v_{z}=v$.

Заметим, что мы не делаем обычного предположения о несжимаемости жидкости, поскольку при больших токах не исключены давления в $10-100 \mathrm{kbar}$ $\left(\approx 10^{9}-10^{10} \mathrm{~Pa}\right)$, когда сжатие струи может быть существенным. Кроме того, в таких приближениях предположение о несжимаемости жидкости $\operatorname{div}(\vec{v})=0$ немедленно приводит к $d v_{z} / d z=0$, т. е. к $v_{z}=$ const, что в силу уравнения неразрывности $v(z) S(z)=$ const приводит к независимости $v$ от $z$.

В итоге, в цилиндрических координатах получается по $\overrightarrow{e_{r}}$ уравнение для радиальной зависимости давления

$$
\frac{1}{\rho} \frac{\partial p}{\partial r}=-\frac{\mu \mu_{0}}{\rho} r j^{2} .
$$

По $\overrightarrow{e_{z}}$ получается уравнение для $v$

$$
v \frac{\partial v}{\partial z}=v \frac{\partial^{2} v}{\partial z^{2}}-\frac{1}{\rho} \frac{\partial p}{\partial z}+g .
$$

Следующим приближением может являться независимость $v_{z}=v$ от радиальной переменной $r$. В самом деле, это - естественное приближение для потока в отсутствии неподвижных стенок, вблизи которых скорость потока должна быть равной нулю. Но тогда давление $p=p(z)$, входящее в правую часть уравнения (3), также должно не зависеть от радиуса, что противоречит уравнению (2) для $p$. Это противоречие легко устранимо, если предположить, что $p$ аналогично $v_{z}$ слабо зависит от $r$. Тогда можно воспользоваться усредненным по $r$ значением $p$ на данной высоте $z$. Интегрируя уравнение $(2)$, получим для $p(z, r)$ :

$$
p(z, r)=-\mu_{0} \mu j^{2} \frac{r^{2}}{2}+C_{1}
$$

где $C_{1}-$ константа интегрирования. В качестве $p(z)$, предполагая слабую зависимость $p(r)$, теперь следует взять среднее значение (от поля на элементе площади $2 \pi r d r)$ по площади $\pi R^{2}(z)=S(z)$. Учтем также условие непрерывности электрического тока $j(z) S(z)=I$ :

$$
p(z)=\frac{1}{\pi R^{2}} \int_{0}^{R} p(z, r) 2 \pi r d r=\frac{\mu_{0} \mu I^{2}}{16 \pi S(z)}+C_{1},
$$

где константа интегрирования, не зависящая от магнитного поля, представляет собой статическое давление $P_{0}$.

C учетом непрерывности потока вещества $\rho v_{0} S_{0}=\rho v(z) S(z) \quad$ (здесь $\quad v_{0} \quad$ и $S_{0}$ соответственно начальные скорость и площадь сечения струи при $z=0)$ уравнение (3) легко решается, хотя решения выражаются через функции Бесселя или функции Эйри. Как следствие, выражения для определения констант интегрирования представляют собой трансцендентные уравнения и могут быть решены только численно. Однако для получения более простых и качественных соотношений между параметрами уместно рассмотреть упрощенную задачу, положив вязкость в уравнении (3) равной нулю. В этом случае оно представляет собой просто дифференциальную форму $(d / d z)$ хорошо известного уравнения Бернулли [4]. При этом давление, создаваемое магнитным полем тока, как легко видеть из (4), совпадает с плотностью энергии магнитного поля, как и должно быть в уравнении Бернулли. Таким образом, условия применимости нашей модели определяются применимостью уравнения Бернулли. Это уравнение, модифицированное с учетом магнитного поля, в итоге можно записать в виде

$$
\rho v^{2}(z) / 2+\rho g z+P_{0}+p(z)=\text { const, }
$$

где $p(z)$ - упоминавшееся давление магнитного поля в сечении на высоте $z$ соответственно.

Определим управляемые параметры данной задачи. Очевидно, в эксперименте можно изменять следующие величины: полный электрический ток $I$, начальное сечение струи, т. е. сечение отверстия $S_{0}$, длину струи $L$ и давление $P_{0}$, которым, очевидно, будет задаваться начальная скорость $v_{0}$. В дальнейшем будем считать управляемым параметром саму скорость $v_{0}$.

Теперь для наших целей, прежде всего, необходимо найти давление $P_{1}$ в конце струи, как функцию управляющих параметров. Для этого используем (4), в которое необходимо подставить площадь сечения в конце струи $S_{1}$, т. е. на заданном расстоянии $L$. Воспользуемся 
для этого уравнением Бернулли (5) в виде

$$
\frac{1}{2} \rho v_{0}^{2}+\rho g L+\frac{\mu_{0} \mu I^{2}}{16 \pi S_{0}}=\frac{\rho v_{0}^{2} S_{0}^{2}}{2 S_{1}^{2}}+\frac{\mu_{0} \mu I^{2}}{16 \pi S_{1}},
$$

где левая часть относится к верхней точке струи, а правая - к нижней; проведена замена $v(L)=v_{0} S_{0} / S_{1}$ из условия непрерывности потока. Отсюда нетрудно найти $S_{1}$ и соответствующее давление $P_{1}$ :

$$
\begin{gathered}
S_{1}=\frac{S_{0}\left(\mu_{0} \mu I^{2} / 16 \pi+\sqrt{A}\right)}{\left(\rho v_{0}^{2} S_{0}+2 \rho g L S_{0}+\mu_{0} \mu I^{2} / 8 \pi\right)}, \\
P_{1}=\frac{\mu_{0} \mu I^{2}\left(\rho v_{0}^{2} S_{0}+2 \rho g L S_{0}+\mu_{0} \mu I^{2} / 8 \pi\right)}{16 \pi S_{0}\left(\mu_{0} \mu I^{2} / 16 \pi+\sqrt{A}\right)},
\end{gathered}
$$

где

$$
\begin{aligned}
A= & \left(\rho^{2} v_{0}^{2}+2 \rho^{2} g L\right) S_{0}^{2} v_{0}^{2} \\
& +\left(\frac{1}{8 \pi} \rho v_{0}^{2} S_{0}+\frac{1}{256 \pi^{2}} \mu_{0} \mu I^{2}\right) \mu_{0} \mu I^{2} .
\end{aligned}
$$

\section{Баланс тепла}

Поскольку наиболее важный рассматриваемый здесь процесс - кристаллизация, то принципиальное значение приобретает распределение температуры в потоке расплава. Рассмотрим температурный режим струи жидкого металла под током. Ясно, что этот режим определяется балансом четырех потоков тепла. Прежде всего, расплав металла находится при температуре не ниже, чем температура плавления и теряет энергию за счет излучения в окружающую среду. В случае достаточно высоких температур (порядка температуры плавления) такие потери могут быть вовсе не малыми. Пренебрегая температурой среды на фоне температуры расплава, для теплоотдачи элемента струи высотой $d z$ и радиуса $R(z)$ в единицу времени можем записать

$$
d Q_{1}=\sigma T^{4} 2 \pi R d z,
$$

где $\sigma$ - постоянная Стефана-Больцмана. Компенсирует эту теплоотдачу тепловыделение тока

$$
d Q_{2}=I^{2} d z / \chi S(z),
$$

где $\chi$ - электропроводность. Следует учесть также два процесса, которые выравнивают распределение температуры вдоль струи: во-первых, „конвекционный“ поток тепла вдоль струи

$$
d Q_{3}=\rho C_{p} \cdot v(z) S(z) d T=\rho C_{p} \cdot v_{0} S_{0} d T,
$$

где мы воспользовались уравнением неразрывности потока вещества; здесь $C_{p}-$ удельная теплоемкость металла. Во-вторых, температура выравнивается за счет обычной диффузии тепла или теплопроводности

$$
d Q_{4}=\kappa \frac{S(z)}{d z} \frac{\Delta T}{d z / L} .
$$

Параметры материалов: температура плавления, плотность, теплопроводность, удельная теплоемкость, электропроводность [6]

\begin{tabular}{c|c|c|c|c|c}
\hline Материал & $T_{m}$ & $\rho$ & $\kappa$ & $\mathrm{Cp}$ & $\chi$ \\
\hline $\begin{array}{c}\text { Единицы } \\
\text { измерения }\end{array}$ & $\mathrm{K}$ & $\mathrm{kg} / \mathrm{m}^{3}$ & $\mathrm{~W} /(\mathrm{m} \cdot \mathrm{K})$ & $\mathrm{J} /(\mathrm{kg} \cdot \mathrm{K})$ & $\Omega \mathrm{m}^{-1} \cdot \mathrm{m}^{-1}$ \\
\hline Сталь & 1790 & 7000 & 47 & 825 & $10^{7}$ \\
\hline Латунь & 950 & 8300 & 100 & 380 & $2.5 \cdot 10^{7}$ \\
\hline Свинец & 600 & 11000 & 35 & 130 & $5 \cdot 10^{6}$
\end{tabular}

Уравнение Фурье для баланса тепла в итоге выглядит следующим образом:

$$
d Q_{4}+d Q_{3}+d Q_{2}-d Q_{1}=0 .
$$

В таблице приведены используемые ниже параметры материалов. Оценки вкладов всех составляющих уравнения баланса тепла, проведенные для всех указанных материалов (с учетом постоянной Стефана-Больцмана $\sigma=5.6710^{-8} \mathrm{~W} /\left(\mathrm{m}^{2} \cdot \mathrm{K}^{4}\right)$ и магнитной проницаемости большинства металлов в расплаве $\mu \approx 1$ ) и в широком диапазоне выбранных для оценок условий эксперимента (в частности, $I=1-1000 \AA, v_{0}=0.1-100 \mathrm{~m} / \mathrm{s}$, $\Delta T=100 \mathrm{~K}, d z=0.001 \mathrm{~m}, L=0.01-1 \mathrm{~m})$, показывают, что поток тепла за счет теплопроводности $Q_{4}$ для всех материалов оказывается на несколько порядков меньше остальных. Таким образом, можно пренебречь вкладом теплопроводности на фоне тепловыделения тока и потерь на излучение.

Тогда уравнение баланса тепла (деленное на $d z$ ) принимает вид

$$
\frac{\Delta T}{\Delta z}=\frac{1}{\rho C_{p} v_{0} S_{0}}\left[\frac{I_{0}^{2}}{\chi S(z)}-2 \pi \sigma R(z) T^{4}(z)\right] .
$$

Оно не имеет аналитического решения, но его можно численно проинтегрировать, явно определив функции $S(z)$ и $R(z)$ из предыдущих уравнений. Однако для нашей цели - выяснения возможности управляемой кристаллизации в потоке расплава - это не является необходимым.

Заметим, что поток тепла $Q_{3}$, связанный с движением расплава, выполняет стабилизирующую функцию. Если температура в нижней точке струи ниже, чем температура в верхнем резервуаре расплава, то поток тепла положительный и происходит нагрев. В противном случае этот поток может охлаждать струю. Поскольку величина этого потока определяется, помимо градиента температуры, еще и скоростью струи, то можно управлять этим потоком, меняя начальную скорость $v_{0}$, а значит, компенсировать избыточный нагрев, увеличивая скорость струи. 


\section{Результаты и обсуждение}

Теперь остается выяснить, существует ли диапазон управляющих параметров $\left(v_{0}, I, S_{0}, L\right)$, в котором может происходить управляемая током кристаллизация расплава в конце струи. Для этого оценим величины давления, необходимые для сдвига температуры кристаллизации вверх. Как известно $([1,5])$, для этого требуются довольно высокие давления, порядка сотен kbar. Для стали эмпирическая зависимость может быть выражена как

$$
T_{c r}=T_{m}+\alpha \cdot p,
$$

где $T_{m}$ - температура плавления при обычных условиях; для стали, например, можно взять [6] $T_{m}=1796 \mathrm{~K}$, $\alpha=2.4 \cdot 10^{-8} \mathrm{~Pa} / \mathrm{K}$. Это означает, что для изменения температуры плавления на $1 \mathrm{~K}$ требуется давление около $100 \mathrm{kbar}$.

Для определенности будем считать, что необходимо увеличить температуру кристаллизации на величину $\delta T=1 \mathrm{~K}$, которое достигается, согласно (7), при некотором давлении $P_{\min }=\delta T / \alpha$. Это дает условие на давление $P_{1}>P_{\min }$. Это можно трансформировать в условие на ток $I>I_{\min }$. Тогда $I_{\min }$ будет являться решением уравнения

$$
\delta T / \alpha=P_{1}\left(v_{0}, I=I_{\min }, S_{0}, L\right),
$$

где $P_{1}$ задается выражением (6). Решение этого алгебраического уравнения четвертой степени по $I^{2}$ тривиально, но слишком громоздко, чтобы приводить его здесь. Результат как функция $I_{\min }\left(v_{0}\right)$ для конкретных параметров приведен на рис. 1.

Другое условие следует из баланса тепловых потоков. Если ток, создающий указанное давление, создает тепловой поток $I^{2} d z / \chi S_{1}$, превышающий потери тепла $\sigma T^{4} 2 \pi\left(S_{1} / \pi\right)^{1 / 2} d z$, то, чтобы нагрев был все же меньшим, чем увеличение температуры кристаллизации $\delta T$, следует потребовать, чтобы начальная скорость потока в „конвективном“ члене была больше некоторой минимальной скорости $v_{0}>v_{0 \text { min. }}$. Эта скорость $v_{0 \text { min }}$ определяется условием $\Delta T / \Delta z<\delta T / L$, откуда следует выражение для вычисления $v_{0}$ :

$$
\frac{\delta T}{L}=\frac{1}{\rho C_{p} v_{0} S_{1}}\left(\frac{I^{2}}{\chi S_{1}}-2 \sigma T_{m}^{4} \pi \sqrt{\frac{S_{1}}{\pi}}\right) .
$$

Легко видеть, что если скорость $v_{0}$ считать заданной, то условие $v_{0}>v_{0 \text { min }}$ можно представить как условие на максимальный ток, такой, что заданная скорость потока еще обеспечивает выполнение условия $\Delta T / \Delta z<\delta T / L$. Тогда выражение (8) определяет функцию $I_{\max }\left(v_{0}, S_{0}, L\right)$. Как и выше, результат получается элементарно, но оказывается слишком громоздким, что бы привести его целиком. Расчет тока $I_{\max }$ как функция скорости $v_{0}$ для конкретных остальных параметров также приведен на рис. 1.
Итак, для выяснения возможности описанного механизма кристаллизации построены две функциональные зависимости: во-первых, $I_{\min }$, т. е. минимальный ток, при котором давление уже достаточно для увеличения температуры кристаллизации на величину $\delta T$ и, во-вторых, ток $I_{\max }$ - максимальный ток, при котором вызываемый им нагрев еще компенсируется набегающим потоком более холодного расплава. Области параметров, при которых $I_{\max }>I_{\min }$ очевидно, и есть искомые условия реализации предложенного метода.

Результаты расчета таких областей для случая расплавов стали, латуни и свинца представлены на рис. 1 . В расчетах использовались приведенные выше параметры материалов, и выбраны реалистичные диапазоны управляющих параметров. Как можно видеть, во всех случаях кривая минимально необходимого тока $I_{\min }$ проходит значительно выше, чем кривая максимально допустимого тока $I_{\max }$. Это означает, что нагрев током настолько велик, что его нельзя скомпенсировать увеличением скорости потока, если ток достаточен для повышения температуры кристаллизации хотя бы на $1 \mathrm{~K}$.

Однако в случаях, изображенных на рис. 1 было использовано естественное значение магнитной проницаемости расплавов порядка единицы. Это значение определяется тем, что температура расплава всегда выше точки Кюри даже для магнитных материалов. Тем не менее этим параметром, в принципе, можно управлять. Так, если ввести в расплав добавку ферромагнитного материала в виде микро- или наноразмерного порошка, который имеет и температуру плавления и температуру Кюри выше температуры плавления данного расплава, можно значительно увеличить магнитную проницаемость всего расплава. В этом случае картина кардинально меняется. Например, для латуни с температурой плавления $T_{m} \approx 950 \mathrm{~K}$ таким материалом может быть микро- или наноразмерный порошок железа, имеющего точку Кюри $T_{c} \approx 1043 \mathrm{~K}$ и магнитную проницаемость $\mu \approx 3000-4000$. Результаты для случая латуни, если довести магнитную проницаемость расплава до величины $\mu \approx 900$, представлены на рис. 2 .

Как можно видеть из рис. $2, a$, при довольно реалистичных условиях процесса (диаметр отверстия $1.8 \mathrm{~mm}$, длина струи $1.5 \mathrm{~cm}$ ) при скоростях выше $12 \mathrm{~m} / \mathrm{s}$ и токе, превышающем $2600 \mathrm{~A}$, существует довольно обширная область, где $I_{\max }>I_{\min }$. Рис. $2, b$ и $c$ показывают, как изменяется эта область при изменении остальных управляющих параметров: длины струи $L$ и диаметра отверстия $D$. Из рис. 2, $c$ видно, что увеличение длины струи до $3 \mathrm{~cm}$ при примерно тех же токах требует увеличения начальной скорости $v_{0}$ до величин $>27 \mathrm{~m} / \mathrm{s}$. Увеличение же диаметра отверстия до $2.5 \mathrm{~mm}$ позволяет снизить скорость до $6 \mathrm{~m} / \mathrm{s}$, но требует тока более $3700 \mathrm{~A}$. Таким образом, как видим, существует широкий диапазон практически реалистичных значений параметров, при которых может реализоваться описанный метод $3 D$-печати. Заметим, что пока это только принципиальный результат: все эти значения (тока и скорости) 

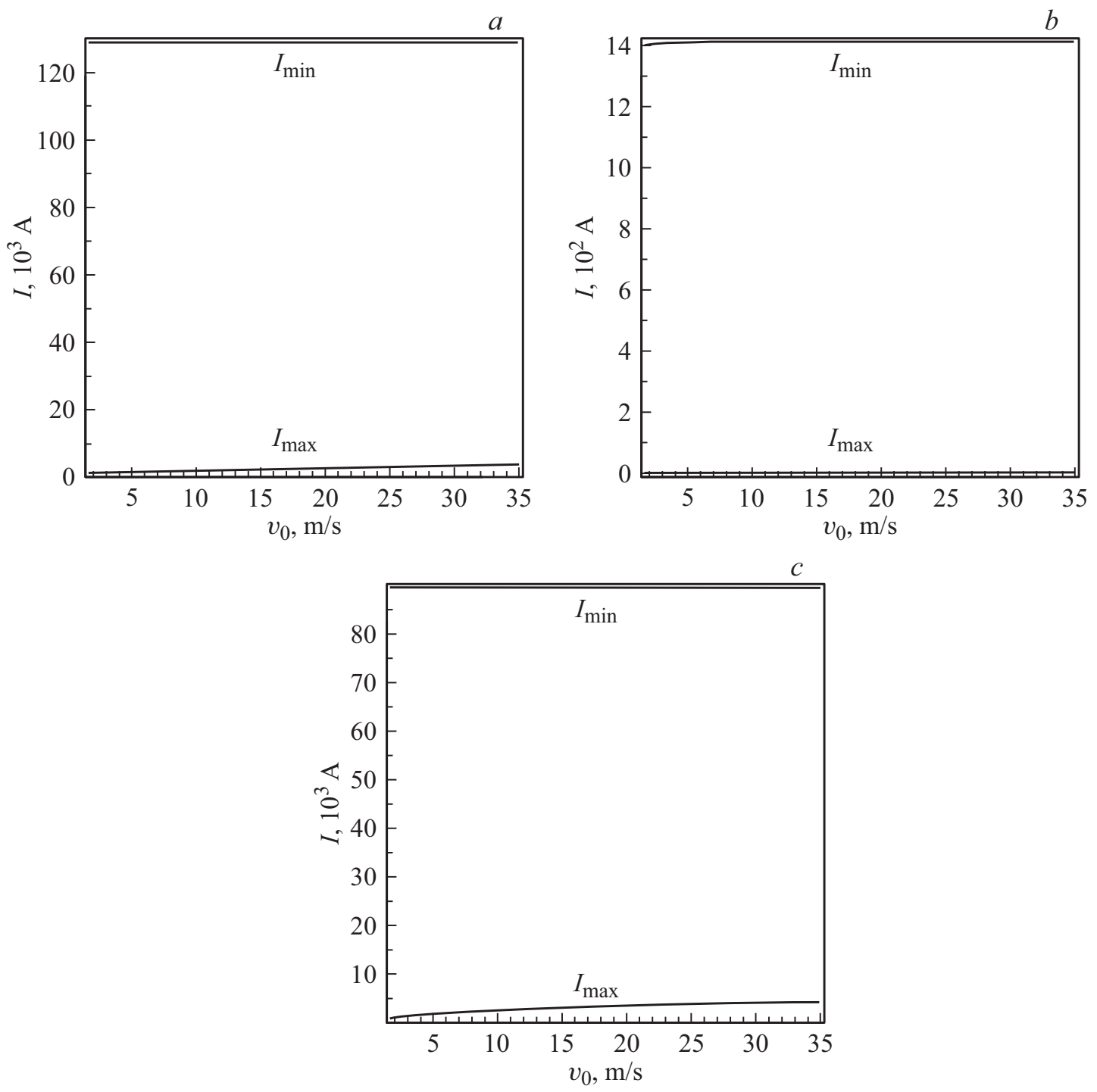

Рис. 1. Область токов $I$ и начальных скоростей $v_{0}$, при которых нагрев за счет омического тепла должен компенсироваться потоком расплава, но ток достаточен для кристаллизации давлением за счет магнитного поля тока $\left(I_{\max }>I_{\min }\right)$ для стали $(a)$, латуни $(b)$ и свинца $(c)$. Как видно, таких областей $I_{\max }>I_{\min }$ нет.

можно существенно уменьшить, увеличив магнитную проницаемость расплава путем большей добавки ферромагнитного материала.

В настоящее время методы создания магнитных жидкостей хорошо разработаны [7,8]. Как правило, такие жидкости получают путем диспергирования магнитного порошка (чаще всего - суспензии наночастиц размером от 10 до $100 \mathrm{~nm}$ ) в немагнитной жидкости. Создание и изучение свойств таких жидкостей в настоящее время являются отдельным направлением исследований. Магнитная восприимчивость таких жидкостей, как хорошо известно, может достигать величин порядка 100-300 единиц (магнитная проницаемость отличается на единицу от магнитной восприимчивости) [7,8]. Строго говоря, такие жидкости обычно не являются ферромагнетиками, поскольку не имеют намагниченности и характерного для ферромагнетиков магнитного гистерезиса.
Скорее их следует считать суперпарамагнетиками [8]. Однако для наших целей ферромагнетизм, очевидно, не требуется. Достаточно лишь величины магнитной проницаемости порядка нескольких сотен единиц. Заметим, что большинство магнитных жидкостей создают на основе органических растворителей [8]. Однако есть работы, в которых показано, что магнитные жидкости нетрудно создать на основе расплава металла: так в работе [9] создана магнитная жидкость на основе расплава палладия с добавкой кобальта, т.е. так, как и требуется для наших целей. Величина магнитной проницаемости при создании магнитной жидкости определяется просто количеством введенного магнитного материала, которое может составлять 25-40\% [9].

Кроме того, дополнительно проведенный расчет показал, что в рамках нашей модели даже при проницаемости около 200 единиц, если уменьшить длину струи 

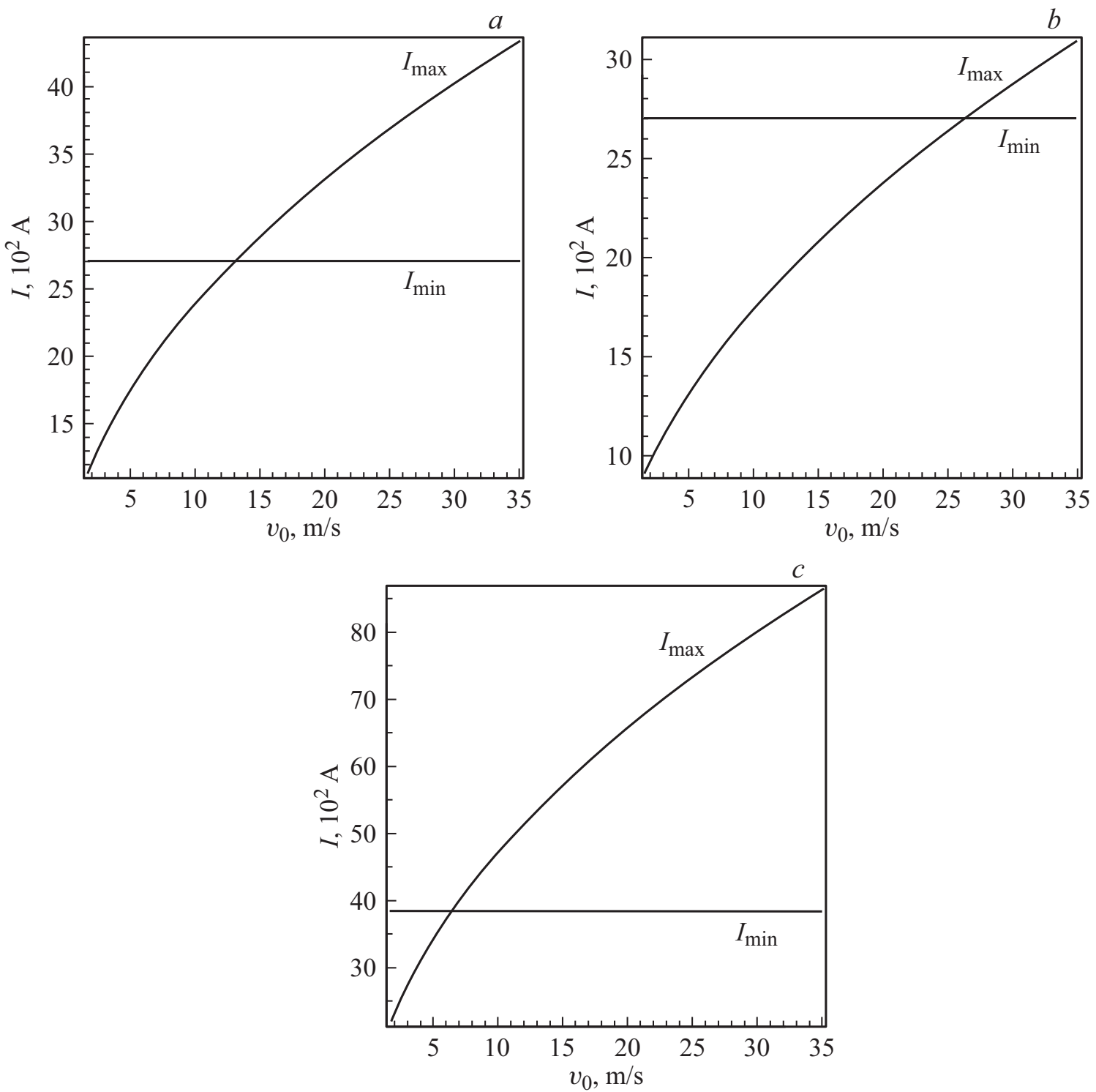

Рис. 2. Область токов $I$ и начальных скоростей $v_{0}$, при которых нагрев за счет омического тепла еще может компенсироваться потоком расплава, но ток достаточен для кристаллизации давлением за счет магнитного поля тока $\left(I_{\max }>I_{\min }\right)$ в латуни при введении ферромагнитной добавки до проницаемости $\approx 900$ при различных остальных управляющих параметрах: $a-$ длина струи $1.8 \mathrm{~cm}$, диаметр отверстия $0.35 \mathrm{~cm} ; b-$ длина струи $5 \mathrm{~cm}$, диаметр отверстия $0.35 \mathrm{~cm} ; c-$ длина струи $1.8 \mathrm{~cm}$, диаметр отверстия $0.5 \mathrm{~cm}$. Как видно, области $I_{\max }>I_{\min }$ существуют.

до $0.5 \mathrm{~cm}$, то характерная диаграмма оказывается такой же, как на рис. 2, $a$ (т.е. существует примерно такая же область, где $\left.I_{\max }>I_{\min }\right)$. Таким образом, достижимость описанного режима в эксперименте выглядит вполне вероятной.

Существует, однако, несколько моментов, вызывающих вопросы. Во-первых, приведенные результаты получены в условиях пренебрежения вязкостью расплава. Может ли вязкость существенно изменить описанную картину? Прежде всего, вязкость может сказаться в ограничении скорости потока через отверстие (так называемый „коэффициент отверстия“ зависит от вязкости). Однако недостаток скорости из-за вязкости всегда можно скомпенсировать за счет повышения давления в исходном резервуаре $P_{0}$. Во-вторых, вязкость расплавов имеет небольшую величину порядка $10^{-6} \mathrm{~Pa} \cdot \mathrm{s}$ и при имеющихся малых изменениях скорости на столь коротком (по отношению к диаметру) пути струи, как можно показать, еще мало сказывается на характерных распределениях скоростей и гидродинамике истечения.

Другим возражением против описанного метода может служить справедливое замечание, что в приведенных расчетах не была учтена скрытая теплота кристаллизации. В самом деле, при кристаллизации выделяется дополнительное тепло, которое может менять картину теплового баланса. Однако простые оценки показывают, 
что в области наших токов это тепло много меньше, чем омический нагрев. Кроме того, избыток тепла всегда можно скомпенсировать выбором $v_{0}$ и $I$ в эксперименте в пределах обозначенного диапазона допустимых параметров.

\section{Заключение}

Таким образом, в работе предложен новый принцип $3 D$-печати для расплава цветных металлов, который дает возможность преодолеть основные проблемы современных аддитивных технологий (низкая скорость печати, малый объем изделия, низкое качество материала изделия). Метод основан на явлении повышения температуры кристаллизации расплава при повышении давления. Давление может создаваться силами Ампера, возникающими при пропускании тока вдоль струи расплава. Построена магнитогидродинамическая модель, показавшая, что прямое осуществление такого принципа невозможно из-за дополнительного нагрева расплава протекающим током. Однако, как оказалось, увеличение магнитной проницаемости расплава до величин порядка сотен единиц (путем введения ферромагнитных добавок, т.е. создание магнитной жидкости известными методами), приводит к появлению широкого диапазона реалистичных параметров, где такой метод индуцированной кристаллизации может реализоваться.

Работа выполнена при поддержке Министерства образования и науки РФ (гос. задание №: 9.1195.2017/4.6).

\section{Список литературы}

[1] Pant M.M. // Phys. Earth and Planetary Interiors. 1978. Vol. 17. N 2. P. $14-15$.

[2] Tillack M.S., Morley N.B. Magnetohydrodynamics Mc Graw Hill, 14th Edition, NY. 1998.

[3] Feynman Lectures of Physics. Vol. 2. Addison Wesley Pablishing Comp., Inc. Reading, Massachusetts, Palo Alto, Lundon. 1964.

[4] White F.M. // Fluid Mechan., Seventh Edition. NY.: Mc Graw-Hill, 2011.

[5] Schlosser H., Vinet P., Ferrante J. // Phys. Rev. B - Condens. Matter. Vol. 40. P. 5929. doi:10.1103/PhysRevB.40.5929

[6] Parker E.R. Materials Data Book for Engineers and Scientists, Mc Graw-Hill, 1967.

[7] Shliomis M.I., Raikher Yu.L. Experimental Investigations of Magnetic Fluids // IEEE Transactions on Magnetic. 1980. Vol. 16. N 2. P. 237-250.

[8] Ferrohydrodynamics (1985), Ronald. E. Rosensweig. The Usual Starting Reference for Learning the Details of Ferrofluids.

[9] Albrecht T., Buhrer C., Fahnle M., Maier K., Platze D., Reske J. // Appl. Phys. A. 1997. Vol. 65. N 2. P. 215-220. (1997) 